\title{
V033 RESERVOIR DESCRIPTION USING ACOUSTIC IMPEDANCE - HALLETTSVILLE 3D
}

\author{
LLOYD R. WEATHERS \\ Mobil E \& P US, 12450 Greenspoint Drive, Houston TX 77060, USA
}

\begin{abstract}
A seismic reservoir prediction methodology generated seismic porosity measurements after conversion of migrated seismic traces to acoustic impedance.
\end{abstract}

Edited well logs were used for wavelet extraction for space adaptive wavelet processing which maximized seismic resolution, corrected the wavelet to zero phase and stabilized residual phase variations spatially.

Each wavelet-processed trace was converted to seismic acoustic impedance by a process that requires minimal $a$ priori information to constrain the acoustic solution. The output is unbiased since it is determined independently trace-by-trace and is not model-driven

Seismic acoustic impedance was calculated independently of well control, but good correlations were still obtained at well ties. Low frequency compensation utilized well logs to map seismic acoustic impedance to absolute acoustic impedance then, by petrophysical calibration, to porosity. Reservoir pore volume (Phi-H) was then calculated from seismic traces and found to show good correlation with well-derived values.

\section{Introduction}

Hallettsville 3-D survey covers 114 square $\mathrm{km}$ in Central Texas, Lavaca County, over Word field, a mature Edwards Cretaceous limestone gas producing field at a depth of $4000 \mathrm{~m}$. Hallettsville 3-D is a CGG speculative vibroseis surver with $25 \mathrm{~m}$ bin spacing. Post migration processing was performed by EnTec Energy Consultants, Ltd. The objective of the borehole calibrated post-migration processing was to maximize resolution, correct to zero phase, compute acoustic impedance and calibrate the seismic data quantitatively to porosity. Figure I shows a portion of a conventionally-processed 3-D migrated dip line through a producing well in Word field. The geologic section is Wilcox, Midway and Navarro siliciclastics from $2.200 \mathrm{~s}$ to the Top of the Austin Chalk which is the strong continuous reflector at $2.736 \mathrm{~s}$ in the well. Below the Austin Chalk is the Edwards limestone top at $2.768 \mathrm{~s}$, which is not a strong reflector. The Edwards limestone at Word field is about $640 \mathrm{~m}$ thick, but economic porosity is developed dominantly in the top $(110 \mathrm{~m})$. The base of this upper Edwards porosity development is readily visible as the strong peak at about $2.800 \mathrm{~s}$. The top of the Edwards is much more difficult to discem as a seismic reflector when it is porous because, in these situations, the Edwards has an acoustic impedance similar to the overlying Austin Chalk. The base of the Edwards formation is clearly evident as the strong trough at $2.980 \mathrm{~s}$.

\section{Well Data Analysis and Log Editing}

Provided that the 3-D volume of stacked, migrated seismic traces conforms to the convolutional model consisting of wavelets convolved with reflectivity series, then wavelets can be estimated at well locations by least squares deconvolution of the seismic traces by the well reflectivity series. However, before using the well acoustic data (sonic and density) to calculate the well reflectivity series, the logs must be properly edited, calibrated and converted to seismic time by means of borehole seismic (check shot or vertical seismic profile). Unreliable logs and inaccurate calibration to borehole seismic are often the cause of unstable and non-convergent extracted wavelets.

Three wells with the most complete data sets were chosen from Word field and logs on these three wells were edited for purposes for wavelet processing and wavelet extraction. Log editing was based on a multi-well approach and was carried out iteratively, with emphasis on testing sonic and density log reliability: Editing was performed only over intervals where it was petrophysically justifiable and was rigorously tested against other $\operatorname{logs}$, other wells and surface seismic data. In the event of discrepancies, the log data is iterated through a data validation and editing cycle until well logs and depth-to-time functions reached optimum reliability with no bias or forced fitting between well and seismic data.

The synthetic seismogram in Figure I was constructed from edited sonic and density logs using the wavelet extracted from the conventional processing.

\section{Space Adaptive Wavelet Processing and Conversion to Acoustic Impedance}

Spatial variation of phase was derived and a spatially adaptive operator was calculated individually for each trace to zero the phase of the estimated wavelet and to normalize the estimated spectrun to a common average spectrum derived from the input data. The wavelet was then spatially stable within the target time window and any residual phase component in the wavelet was estimated using a simultaneous multi-well approach so that the resulting traces are accurately zero phase. A spectral inversion operation on these zero phase traces was then carried out utilizing available bandwidth.

Overall the section in Figure 2 is broader bandwidth, showing greater stratigraphic and structural detail than the conventional processing in Figure I without introducing much noise. As an example of the benefits of broader usable bandividth, the fault in the Austin reflector at 
Trace 142 shows clear offset, which is not resolved in the conventional processing of Figure 1 . The synthetic seismogram in Figure 2 was constructed from the same reflectivity series as in Figure 1, but with the broader band wavelet resulting from the space adaptive wavelet processing.

These broad band wavelet-processed wiggle traces represent band-limited reflectivity between acoustic impedance layers of the earth. Conversion of the wiggle traces to a representation of the bulk properties of the layers themselves was accomplished by an iterative technique that transforms the interfaces to estimates of the laver acoustic impedance. The technique is unbiased since it does not require any a priori information to constrain the solution. Each output acoustic impedance trace is determined independently of the next trace and independently of any well that may be nearby.

\section{Petrophysical Calibration}

For quantitative petrophysical calibration, absolute acoustic impedance is required. Well information was used to insert the information below $6 \mathrm{~Hz}$ missing from seismic traces. A well macro-model was constructed using eight wells. Six structural horizons were used to guide the mapping of well log information on to all seismic traces in the 3-D data volume during low frequency compensation. Figure 3 compares the seismic absolute acoustic impedance estimate to well log acoustic impdedance at each of three well locations. The units of acoustic impedance in Figure 3 is Acoustic Megohms (AMO). One acoustic ohm is in units of $\mathrm{kgs}^{-1} \mathrm{~m}^{-2}$. The post migration seismic analysis and processing produced a good prediction of the layering at each well, both in terms of curve shape as well as in absolute value. For example, seismic acoustic impedance accurately measured the economic porosity between Top of Edwards and Base of Edwards Porosity in the well on the left (WW-1).

Absolute acoustic impedance was transformed to Edwards limestone porosity units using an empirically derived relationship calibrated to well porosity measurements. The effect of gas saturation in this highly indurated Cretaceous limestone can be ignored because it has little effect on the overall acoustic properties of the limestone. The calculated seismic acoustic porosity resulted in a data volume in addition to the others available: relative acoustic impedance, space adaptive wavelet processing and the original 3D migrated data volume. This additional seismic porosity data volume has a wide range of reservoir description applications ranging from studies of reservoir compartmentalization to reservoir volumetrics.

In order to address reservoir volumetrics, a transform between acoustic impedance and velocity was used to convert absolute acoustic impdeance to velocity, sample- by-sample, and another data volume produced: Edwards limestone seismic porosity sampled at 3 meter interval rather than $2 \mathrm{~ms}$.

Having both thickness and porosity, it was then possible to sum porosity values to generate total available net pore space, Phi-H, (porosity-meters or prosity-feet) within the reservoir.

Elongate trends of high seismic $\mathrm{Phi}-\mathrm{H}$ that are very apparant in map vieiv are consistent with a geologic model of meteoric water induced porosity enhancement in backreef shoaling, but the level of detail and the imaging of lateral inhomogeneity provided by seismic $\mathrm{Phi}-\mathrm{H}$ generated a new depth of understanding of the field. Figure 4 shows how the volumetrics (Reservoir Net Phi-H) at a seismic trace at each of nine well locations matches the actual well $\log$ Phi-H. There is good correlation between well log porosity-thickness and seismic porosity-thickness at the well locations.

\section{Conclusions}

Bore-hole calibrated post-migration data analysis and processing met its objective of maximizing the resolution of wiggle trace seismic data, correcting the data accurately to zero phase and producing acoustic impedance as an alternative to wiggle trace reflectivity. Since, for the Edwards, there is a direct relationship between acoustic impedance and effective reservoir porosity, it was also possible to petrophysically calibrate seismic data. Reservoir porosity calculated from seismic allowed the imaging of very complicated lateral reservoir inhomogeneity with the full lateral resolution afforded by seismic data, not limited by density of available well control. This case history shows that it is possible to calculate quantitative petrophysical measurements from seismic traces by advanced techniques while maintaining connection back to the physics of the original measurements. The first successful horizontal well in the Edwards formation has been drilled by Mobil in Word field, incorporating this seismic porosity estimation technology.

\section{Acknowledgment}

Thanks to CGG American Services for permission to show the seismic data, to EnTec Energy Consultants, a wholely owned subsidiary of Western Atlas International, for the post-migration reservoir description data analysis and processing and to Mobil E \& P US, Inc. for permission to show the results. 


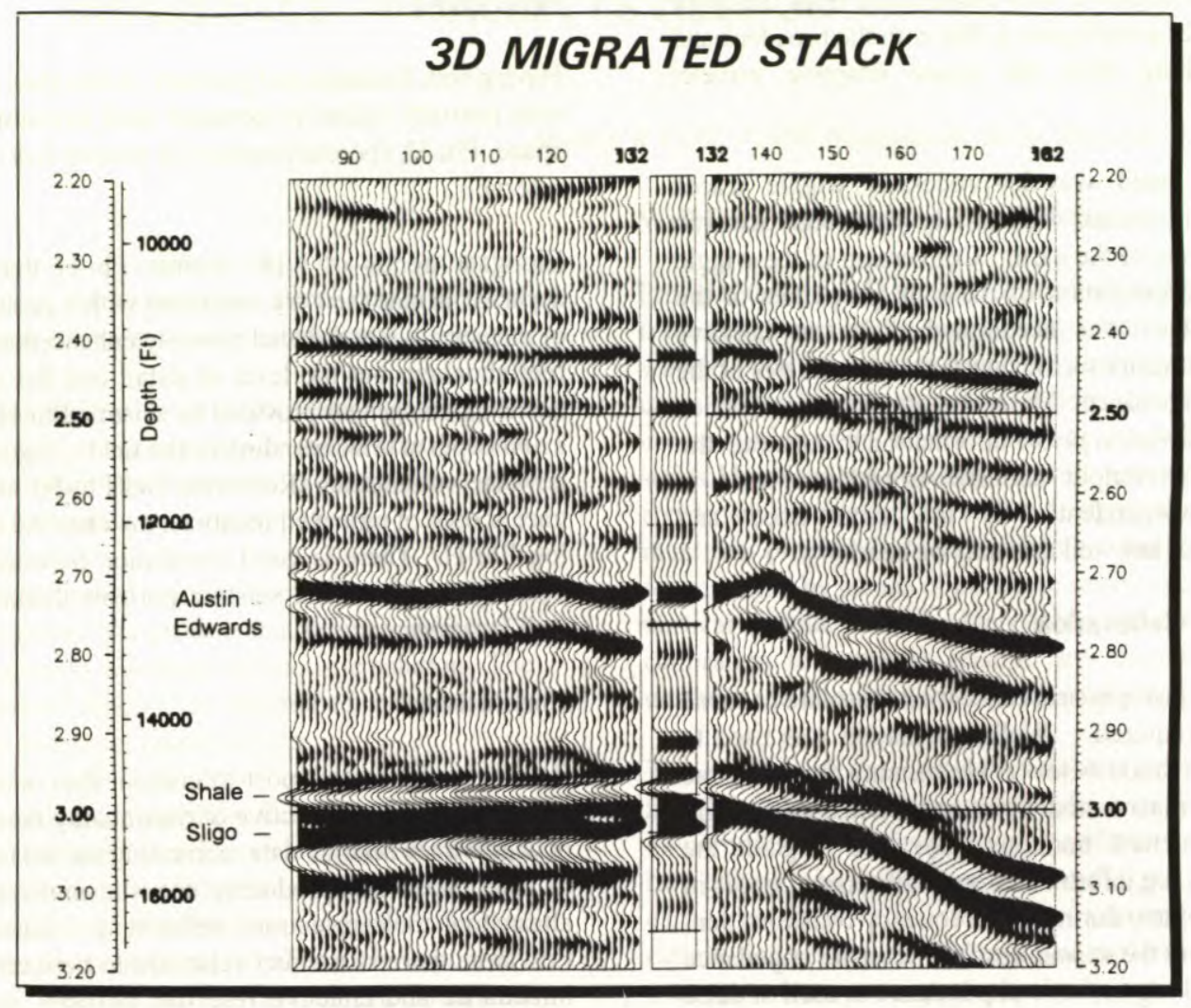

Fig. 1. 3-D Migrated stack with conventional processing.

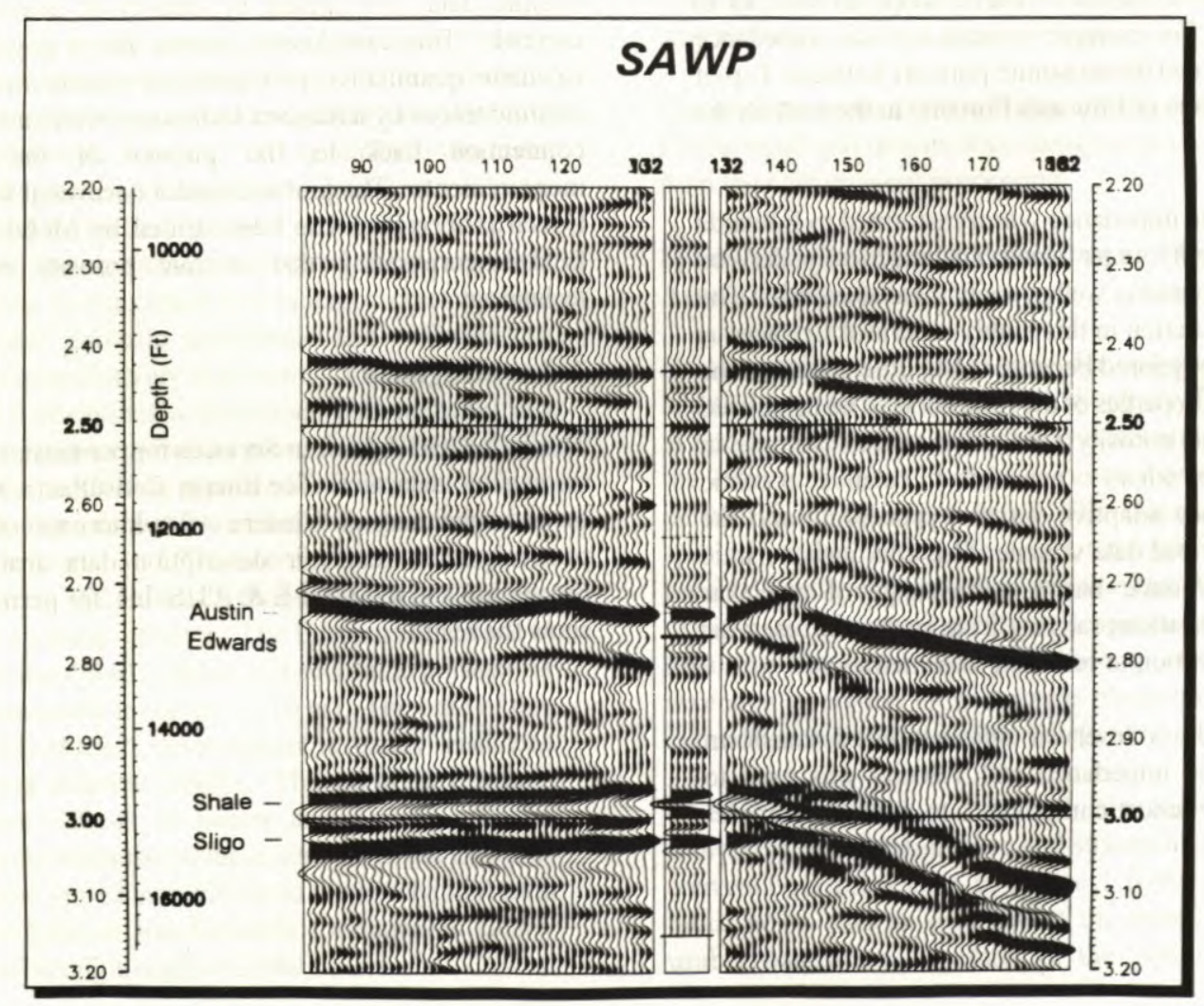

Fig. 2. Space adaptive wavelet processing. 


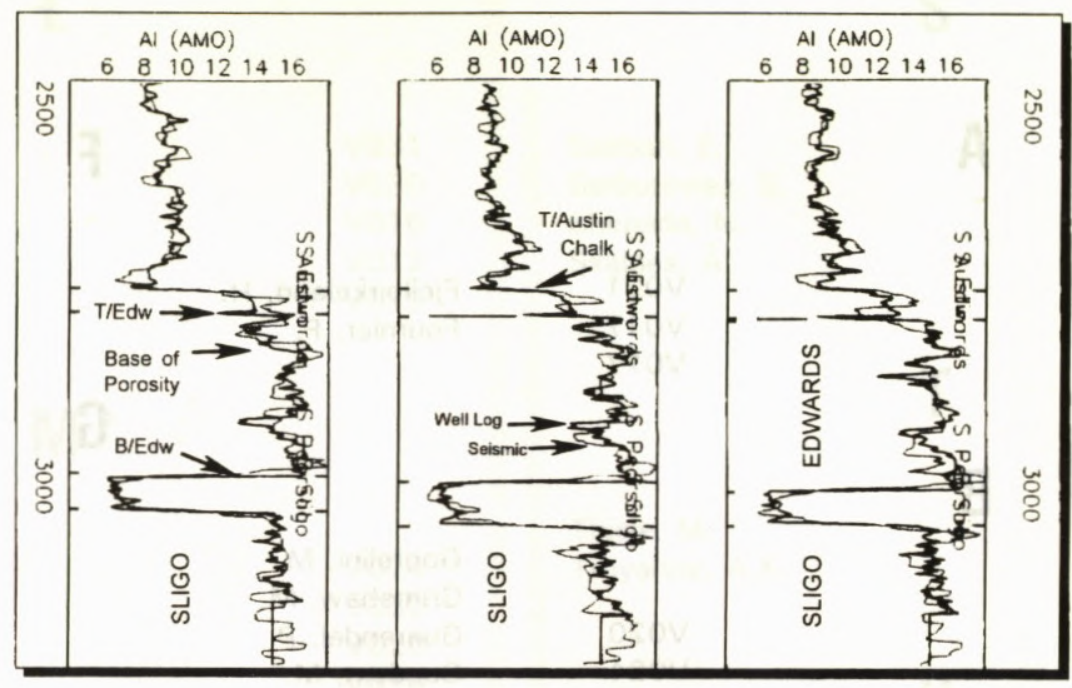

Fig. 3. Absolute seismic acoustic impedance compared to well log acoustic impedance.

\begin{tabular}{|ccc|}
\hline Well & $\begin{array}{c}\text { Seismic } \\
\text { Trace }\end{array}$ & $\begin{array}{c}\text { Well } \\
\text { Log }\end{array}$ \\
\hline$W W-12$ & 18 & 26 \\
$W W-8$ & 16 & 19 \\
$W W-1$ & 15 & 19 \\
$W W-2$ & 7 & 12 \\
$W W-11$ & 3 & 5 \\
$W W-9$ & 2 & 3 \\
$W W-7$ & 3 & 3 \\
$W W-10$ & 2 & 3 \\
$W W-3$ & 2 & 1 \\
& & \\
\hline
\end{tabular}

Fig. 4. Reservoir net Phi-H (ft) Volumetrics (Porosity-ft)

Well log compared to seismic. 